\title{
MRI-based Algorithm for Acute Ischemic Stroke Subtype Classification
}

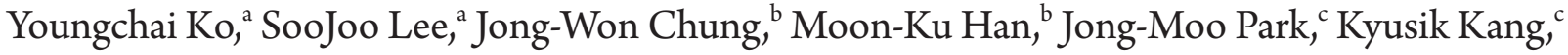 \\ Tai Hwan Park, ${ }^{\mathrm{d}}$ Sang-Soon Park, ${ }^{\mathrm{d}}$ Yong-Jin Cho, ${ }^{\mathrm{e}}$ Keun-Sik Hong, ${ }^{\mathrm{e}}$ Kyung Bok Lee, ${ }^{\mathrm{f}}$ Jun Lee, ${ }^{\mathrm{g}}$ \\ Dong-Eog Kim, ${ }^{\mathrm{h}}$ Dae-Hyun Kim, ${ }^{\mathrm{i} J a e-K w a n ~ C h a, ~}{ }^{\mathrm{i}}$ Joon-Tae Kim, ${ }^{\mathrm{j}}$ Jay Chol Choi, ${ }^{\mathrm{k}}$ Dong-Ick Shin, ${ }^{1}$ \\ Ji Sung Lee, ${ }^{\mathrm{m}}$ Juneyoung Lee, ${ }^{\mathrm{n}}$ Kyung-Ho Yu, ${ }^{\circ}$ Byung-Chul Lee, ${ }^{\circ}$ Hee-Joon Bae ${ }^{\mathrm{b}}$ \\ ${ }^{a}$ Department of Neurology, Eulji University Hospital, Eulji University, Daejeon, Korea \\ ${ }^{b}$ Department of Neurology, Cerebrovascular Center, Seoul National University Bundang Hospital, Seongnam, Korea \\ 'Department of Neurology, Eulji General Hospital, Eulji University, Seoul, Korea \\ ${ }^{\mathrm{d}}$ Department of Neurology, Seoul Medical Center, Seoul, Korea \\ e Department of Neurology, Ilsan Paik Hospital, Inje University, Goyang, Korea \\ ${ }^{\mathrm{f}}$ Department of Neurology, Soonchunhyang University Hospital, Seoul, Korea \\ ${ }^{g}$ Department of Neurology, Yeungnam University Hospital, Daegu, Korea \\ ${ }^{\mathrm{h}}$ Department of Neurology, Dongguk University Ilsan Hospital, Goyang, Korea \\ iDepartment of Neurology, Dong-A University Hospital, Busan, Korea \\ 'Department of Neurology, Chonnam National University Hospital, Gwangju, Korea \\ ${ }^{k}$ Department of Neurology, Jeju National University Hospital, Jeju National University School of Medicine, Jeju, Korea \\ ${ }^{1}$ Department of Neurology, Chungbuk National University College of Medicine, Cheongju, Korea \\ mBiostatistical Consulting Unit, Soonchunhyang University Medical Center, Seoul, Korea \\ ${ }^{\mathrm{n}}$ Department of Biostatistics, Korea University College of Medicine, Seoul, Korea \\ ${ }^{\circ}$ Department of Neurology, Hallym University Sacred Heart Hospital, Anyang, Korea
}

Background and Purpose In order to improve inter-rater reliability and minimize diagnosis of undetermined etiology for stroke subtype classification, using a stroke registry, we developed and implemented a magnetic resonance imaging (MRI)-based algorithm for acute ischemic stroke subtype classification (MAGIC).

Methods We enrolled patients who experienced an acute ischemic stroke, were hospitalized in the 14 participating centers within 7 days of onset, and had relevant lesions on MR-diffusion weighted imaging (DWI). MAGIC was designed to reflect recent advances in stroke imaging and thrombolytic therapy. The inter-rater reliability was compared with and without MAGIC to classify the Trial of Org 10172 in Acute Stroke Treatment (TOAST) of each stroke patient. MAGIC was then applied to all stroke patients hospitalized since July 2011, and information about stroke subtypes, other clinical characteristics, and stroke recurrence was collected via a web-based registry database.

Results The overall intra-class correlation coefficient (ICC) value was $0.43(95 \% \mathrm{Cl}, 0.31$ $0.57)$ for MAGIC and $0.28\left(95 \% \mathrm{Cl}_{1} 0.18-0.42\right)$ for TOAST. Large artery atherosclerosis (LAA) was the most common cause of acute ischemic stroke (38.3\%), followed by cardioembolism (CE, 22.8\%), undetermined cause (UD, 22.2\%), and small-vessel occlusion (SVO, 14.6\%). Oneyear stroke recurrence rates were the highest for two or more UDs $(11.80 \%)$, followed by LAA (7.30\%), CE (5.60\%), and SVO (2.50\%).

Conclusions Despite several limitations, this study shows that the MAGIC system is feasible and may be helpful to classify stroke subtype in the clinic.
Correspondence: Hee-Joon Bae Department of Neurology, Cerebrovascular Center, Seoul National University Bundang Hospital, 82 Gumi-ro 173-Beon gil, Bundang-gu, Seongnam 463-707, Korea Tel: +82-31-787-7467 Fax: +82-31-787-4563 E-mail:braindoc@snu.ac.kr

Received: June 25, 2014 Revised: July 30, 2014 Accepted: July 30, 2014

This study was supported by grant No. 02-2006-041 from the SNUBH Research Fund and a grant of the Korea Healthcare technology RetD Project, Ministry of Health and Welfare, Republic of Korea (HI10C2020).

The authors have no financial conflicts of interest.

Keywords Stroke; Magnetic resonance imaging; Algorithm; Classification 


\section{Introduction}

Stroke is a heterogeneous neurological disorder, and it is clear that functional outcome, recurrence, and strategies for secondary prevention differ by subtype. ${ }^{1}$ To enable robust research in these areas, accurate classification of different stroke syndromes into homogenous subgroups is important. ${ }^{2}$

The Trial of Org 10172 in Acute Stroke Treatment (TOAST) investigators devised a classification system of ischemic stroke subtypes based on inferred cause, ${ }^{3}$ which has gained wide acceptance for both clinical and research purposes. ${ }^{2}$ However, in a series of 18 patients who were independently assessed by 24 physician-investigators, the scheme had moderate inter-rater reliability (overall $\kappa=0.54)^{4}$ mainly because of inflating the category of "stroke of undetermined etiology." Depending on the quality, completeness, and rapidity of the work-up, most registries that use the TOAST system for stroke classification have failed to identify a definite cause in $25 \%-39 \%$ of patients. ${ }^{5}$

Recent advances in stroke imaging (diffusion weighted imaging, DWI; high-resolution wall imaging) and evaluation of embolic sources makes it possible to develop criteria for the most likely mechanism behind the ischemic stroke. ${ }^{1,6}$ Refining algorithms for determining the subtype of ischemic stroke enables physicians to make a consistent decision on the diagnosis of the stroke subtype and improve inter-rater agreement. ${ }^{3}$ In this study, in an attempt to improve inter-rater reliability of the TOAST system and to minimize the proportion of undetermined stroke, we developed and validated the magnetic resonance imaging (MRI)-based diagnostic algorithm for acute ischemic stroke subtype classification (MAGIC) that reflects recent advances in stroke imaging and thrombolytic therapy. We describe and compare baseline characteristics, extent of diagnostic evaluation, and acute management and stroke recurrence of our stroke population according to stroke subtypes diagnosed by this algorithm.

\section{Methods}

\section{Study population}

This study was performed based on a prospective stroke registry of the fifth section of the Clinical Research Center for Stroke (CRCS-5). ${ }^{7}$ The CRCS-5 was sponsored by the Korean government and began to collect data from hospitalized patients with stroke in order to characterize the epidemiology of stroke and the status of stroke care in Korea. Among the patients who were hospitalized in the 14 participating centers between July 2011 and May 2013, those who met all of the following eligibility criteria were enrolled in this study: 1) diagnosed ischemic stroke; 2) arrived at a hospital within 7 days of onset; and 3) had relevant lesions on DWI.

Information on demographics, medical history, risk factors, stroke characteristics, including TOAST classification using MAGIC, and acute management were obtained directly from the registry database of the CRCS-5.

In all participating centers, approval was obtained from local institutional review boards for collection of anonymized clinical data without patients' consent into the registry database from 2008 to monitor and improve the quality of stroke care. In 2011, we got further approval from institutional review boards for collecting the outcomes for hospitalized patients with informed consent from them or their family members.

\section{Algorithm development}

MAGIC was first proposed in 2008 and was revised during the subsequent two years through monthly steering committee meetings of the CRCS-5. For training on MAGIC, we developed a website (www.crcsmagic.com). All the neurology residents, stroke physicians, and stroke nurses participating in the CRCS5 were requested to train themselves through this website.

MAGIC is composed of the five following steps: 1) consideration of other determined etiology of stroke; 2) screening for small-vessel occlusion (SVO) on DWI; 3 ) consideration of relevant artery stenosis or occlusion; 4) consideration of recanalization status after thrombolytic therapy; and 5) consideration of follow-up recanalization status without thrombolytic therapy. The order of steps and other details of MAGIC were designed for improving the feasibility and convenience in applying the algorithm in clinical practice and were finalized after feedback from participating stroke physicians.

\section{Step 1. Consideration of other determined etiology of stroke (Figure $1 A$ )}

The other causes category includes patients with a diverse array of stroke mechanisms. Disorders included in this category are difficult to categorize into more homogenous groups. A patient who has a rare cause of ischemic stroke (Supplementary Table 1$)^{1}$ would be classified as "other determined cause (OD)" or "two or more undetermined causes ( $\mathrm{UD} \geq 2)$ ), according to coexistence of other stroke etiology such as large artery atherosclerosis (LAA), SVO, and cardioembolism (CE).

\section{Step 2. Screening for SVO using DWI (Figure 1B)}

A single lesion with the largest diameter of $\leq 20 \mathrm{~mm}$ in an axial slice of DWI for penetrating artery infarction of the basal ganglia, corona radiate, thalamus, or pons would be classified as a SVO. ${ }^{1}$ If medical history or electrocardiography (ECG) identifies high-risk cardioembolic sources (Supplementary Table 2), ${ }^{1}$ 


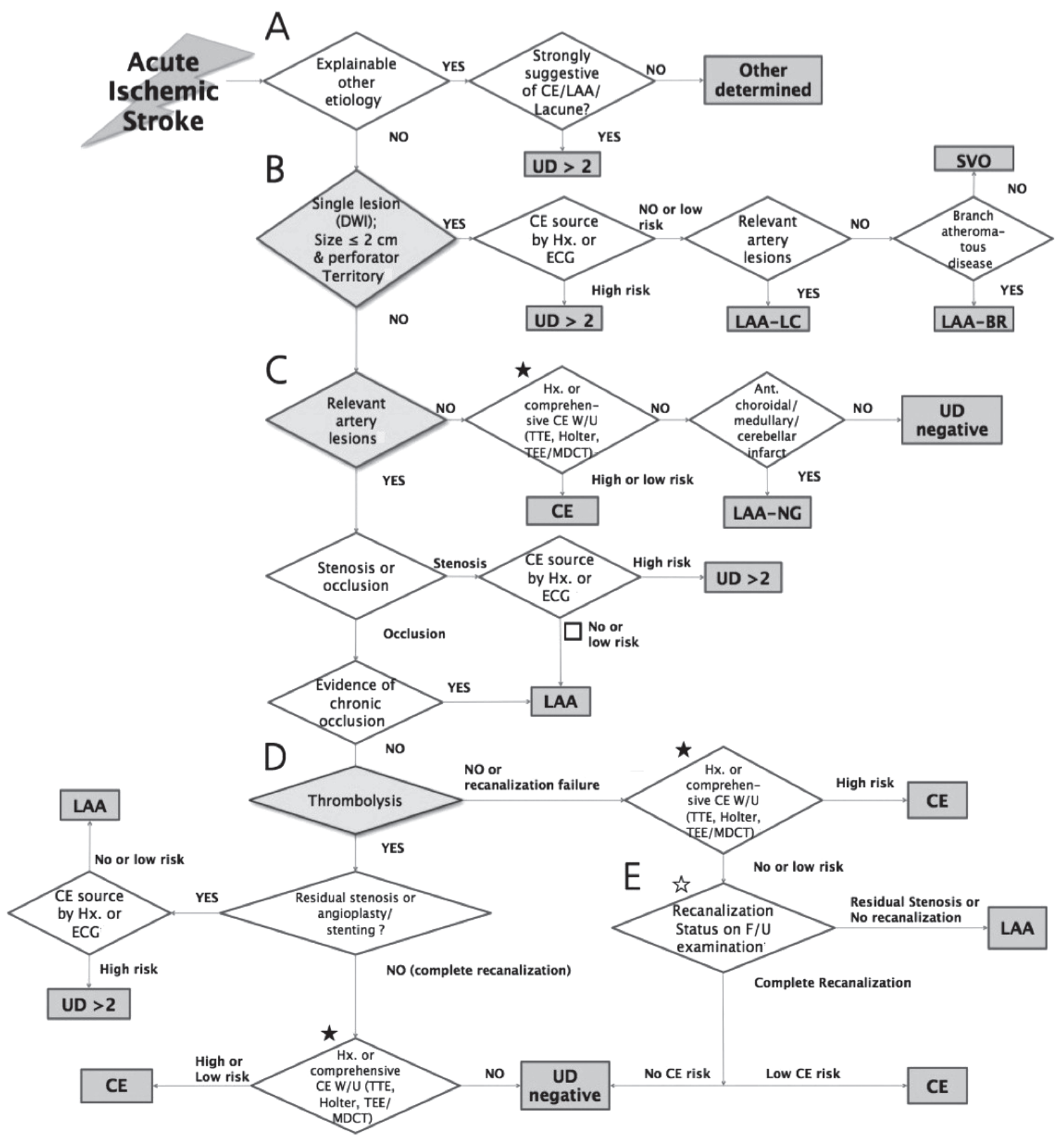

Figure 1. MRI-based algorithm for acute ischemic stroke classification (MAGIC) (A) Step 1: Consideration of other determined etiology of stroke. (B) Step 2: Screening for small vessel occlusion (SVO) using MRI. (C) Step 3: Consideration of relevant artery stenosis or occlusion. (D) Step 4: Consideration of recanalization status of occluded artery after thrombolytic therapy. (E) Step 5: Consideration of follow-up recanalization status of occluded artery without thrombolytic therapy. $\star$ : If one of three examinations (TTE, Holter, and TEE [or MDCT]) was not performed, then the patient was classified as 'undetermined incomplete'. 败: The follow-up vascular status would be evaluated by MR/CT angiography or transcranial doppler (TCD). If no examinations are performed, then the patient should be classified as 'undetermined incomplete'. LAA, large artery atherosclerosis; SVO, small vessel occlusion; CE, cardioembolism; UD, undetermined cause; UD $\geq 2$, two or more undetermined causes; DWI, diffusion weighted image; Hx, history; ECG, electrocardiography; LAA-LC, large artery atherosclerosis with lacunae; LAA-BR, branch atheromatous disease; W/U, work-up; TTE, transthoracic echocardiography; TEE, transesophageal echocardiography; MDCT, multi-detector row computerized tomography; Ant, Anterior; LAA-NG, large artery atherosclerosis with normal angiography; F/U, follow-up.

then that infarction is classified as UD $\geq 2$. If accompanied by relevant stenosis of a corresponding cerebral artery on angiographic evaluation, including CT (computerized tomography) angiography, MR angiography, or conventional angiography, then it is classified as a "LAA-LC (large artery atherosclerosis with lacunae)". Infarctions in the midline extending from the base of the pons into the tegmentum without significant relevant artery stenosis would be classified as "branch atheroma- 
tous disease (LAA-BR)."

\section{Step 3. Consideration of relevant artery stenosis or occlusion (Figure 1C)}

Relevant arterial pathology was defined as stenosis or occlusion of arteries supplying the vascular territory of acute ischemic lesions detected on DWI. Stenosis less than 50\% was also regarded as being relevant when clinical syndromes, lesions patterns on DWI, and new imaging techniques such as high-resolution wall imaging supported its relevance.

In cases of a single lesion with the largest diameter $>20 \mathrm{~mm}$ or multiple lesions with no steno-occlusion of relevant artery on angiographic evaluation, a possibility of cardioembolic stroke should be considered. "Extensive embolic source evaluation", including 24-h Holter monitoring (24-h Holter), transthoracic echocardiography (TTE), and transesophageal echocardiography (TEE) are recommended. In some uncooperative patients, cardiac multi-detector computed tomography $(\mathrm{MDCT})^{8}$ substitutes for TEE. Infarctions in which a definite cardioembolic source is not revealed in spite of a comprehensive work-up would be classified as "undetermined negative (UD-negative)". However, when relevant lesions are located at the anterior choroidal artery territory, single territory of cerebellum, or medullar oblongata, where SVOs do not seem to be causing infarctions, traditional MRI techniques cannot detect vascular pathologies of a relevant artery, and atherosclerosis may be a dominant vascular pathology, ${ }^{9-12}$ the infarctions are classified as "LAA-NG (large artery atherosclerosis with normal angiography)" instead of "UD-negative".

When a relevant pathology of a corresponding artery is observed, it is divided into stenosis and occlusion. If medical his-

Table 1. Baseline characteristics, according to stroke subtype

\begin{tabular}{|c|c|c|c|c|c|c|c|c|}
\hline & LAA & SVO & CE & OD & $\begin{array}{c}\text { UD } \\
\text { two or more }\end{array}$ & $\begin{array}{c}\text { UD } \\
\text { negative }\end{array}$ & $\begin{array}{c}\text { UD } \\
\text { incomplete }\end{array}$ & $P$ value \\
\hline Number of patients & $2,541(38.3)$ & $964(14.6)$ & $1,508(22.8)$ & $138(2.1)$ & $320(4.8)$ & $457(6.9)$ & $696(10.5)$ & \\
\hline Male & $1,563(61.5)$ & $599(62.1)$ & $792(52.5)$ & $81(58.7)$ & $183(57.2)$ & $297(65.0)$ & $404(58.0)$ & $<0.001^{*}$ \\
\hline Age (years), mean \pm SD & $67.7 \pm 12.2$ & $64.0 \pm 12.4$ & $71.9 \pm 11.6$ & $52.4 \pm 16.4$ & $72.4 \pm 11.0$ & $65.2 \pm 13.0$ & $69.4 \pm 12.6$ & $<0.001^{\dagger}$ \\
\hline FAT to arrival (h), median (IOR) & $12(3-43)$ & $14(4-41)$ & $3(1-11)$ & $11(1-48)$ & $5(1-25)$ & $6(1-36)$ & $7(2-33)$ & $<0.001^{\ddagger}$ \\
\hline Initial NIHSS, median (IOR) & $4(2-7)$ & $2(1-4)$ & $8(2-15)$ & $2(1-6)$ & $5(2-12)$ & $3(1-7)$ & $5(2-12)$ & $<0.001^{\ddagger}$ \\
\hline Previous mRS & & & & & & & & $<0.001^{*}$ \\
\hline 0 & 1,998 (78.6) & 808 (83.8) & $1,159(76.9)$ & $110(79.7)$ & $245(76.6)$ & $389(85.1)$ & $513(73.7)$ & \\
\hline $1-5$ & $543(21.4)$ & $156(16.2)$ & $349(23.1)$ & $28(20.3)$ & $75(23.4)$ & $68(14.9)$ & $183(26.3)$ & \\
\hline History of stroke & $575(22.6)$ & $152(15.8)$ & $355(23.5)$ & $31(22.5)$ & $87(27.2)$ & $77(16.8)$ & $127(18.2)$ & $<0.001^{*}$ \\
\hline Hypertension & $1,790(70.4)$ & $628(65.1)$ & $1,034(68.6)$ & $69(50.0)$ & $248(77.5)$ & $266(58.2)$ & $428(61.5)$ & $<0.001^{*}$ \\
\hline Diabetes mellitus & $975(38.4)$ & $322(33.4)$ & $439(29.1)$ & $22(15.9)$ & $105(32.8)$ & $111(24.3)$ & $196(28.2)$ & $<0.001^{*}$ \\
\hline Hyperlipidemia & $865(34.0)$ & 307 (31.8) & $419(27.8)$ & $26(18.8)$ & $87(27.2)$ & $163(35.7)$ & $160(23.0)$ & $<0.001^{*}$ \\
\hline Coronary artery disease & $172(6.8)$ & $50(5.2)$ & $180(11.9)$ & $4(2.9)$ & $43(13.4)$ & $38(8.3)$ & $47(6.8)$ & $<0.001^{*}$ \\
\hline Current smoking & $1,070(42.1)$ & $455(47.2)$ & $461(30.6)$ & $53(38.4)$ & $107(33.4)$ & $195(42.7)$ & $269(38.6)$ & $<0.001^{*}$ \\
\hline Atrial fibrillation & $10(0.4)$ & $1(0.1)$ & $1175(77.9)$ & $1(0.7)$ & $265(82.8)$ & $0(0)$ & $20(2.9)$ & $<0.001^{*}$ \\
\hline Total cholesterol (mg/dL), mean \pm SD & $200.1 \pm 131.5$ & $191.4 \pm 95.3$ & $183.2 \pm 127.1$ & $221.5 \pm 209.8$ & $189.4 \pm 127.9$ & $192.0 \pm 115.2$ & $199.3 \pm 141.0$ & $<0.001^{\dagger}$ \\
\hline $\mathrm{FBS}(\mathrm{mg} / \mathrm{dL})$, mean $\pm \mathrm{SD}$ & $163.3 \pm 190.9$ & $149.6 \pm 171.7$ & $176.3 \pm 217.7$ & $178.0 \pm 234.6$ & $170.7 \pm 207.0$ & $147.6 \pm 181.3$ & $160.7 \pm 171.2$ & $0.014^{\dagger}$ \\
\hline $\mathrm{SBP}(\mathrm{mmHg})$, mean $\pm \mathrm{SD}$ & $141.7 \pm 31.5$ & $154.8 \pm 29.4$ & $143.2 \pm 34.2$ & $139.3 \pm 22.2$ & $147.7 \pm 28.1$ & $144.6 \pm 26.0$ & $144.1 \pm 41.5$ & $<0.001^{\dagger}$ \\
\hline $\mathrm{DBP}(\mathrm{mmHg})$, mean $\pm \mathrm{SD}$ & $85.2 \pm 23.7$ & $90.5 \pm 17.2$ & $84.5 \pm 28.8$ & $81.8 \pm 13.5$ & $85.6 \pm 18.0$ & $85.3 \pm 15.1$ & $84.6 \pm 37.9$ & $<0.001^{\dagger}$ \\
\hline Thrombolysis & & & & & & & & $<0.001^{*}$ \\
\hline IV & $188(7.4)$ & $49(5.1)$ & $241(16.0)$ & $8(5.8)$ & $47(14.7)$ & $46(10.1)$ & $67(9.6)$ & \\
\hline $\mid A$ & $64(2.5)$ & $0(0)$ & $81(5.4)$ & $1(0.7)$ & $11(3.4)$ & $15(3.3)$ & $11(1.6)$ & \\
\hline $\mid \mathrm{V}+\mathrm{IA}$ & $61(2.4)$ & $0(0)$ & $136(9.0)$ & $4(2.9)$ & $13(4.1)$ & $26(5.7)$ & $23(3.3)$ & \\
\hline \multicolumn{9}{|l|}{ Discharge medication } \\
\hline Aspirin & $2,183(85.9)$ & $838(86.9)$ & $545(36.1)$ & $86(62.3)$ & $149(46.6)$ & $356(77.9)$ & $523(75.1)$ & $<0.001^{*}$ \\
\hline Clopidogrel & $1,289(50.7)$ & $276(28.6)$ & $181(12.0)$ & $43(31.2)$ & $74(23.1)$ & $165(36.1)$ & $215(30.9)$ & $<0.001^{*}$ \\
\hline Cilostazol & $281(11.1)$ & $122(12.7)$ & $42(2.8)$ & $11(8.0)$ & $18(5.6)$ & $24(5.3)$ & $66(9.5)$ & $<0.001^{*}$ \\
\hline Triflusal & $25(1.0)$ & $11(1.1)$ & $15(1.0)$ & $2(1.4)$ & $6(1.9)$ & $1(0.2)$ & $5(0.7)$ & $0.377^{*}$ \\
\hline Ticlopidine & $46(1.8)$ & $7(0.7)$ & $9(0.6)$ & $0(0)$ & $1(0.3)$ & $4(0.9)$ & $11(1.6)$ & $0.003^{*}$ \\
\hline Others antiplatelet & $5(0.2)$ & $3(0.3)$ & $5(0.3)$ & $1(0.7)$ & $2(0.6)$ & $1(0.2)$ & $2(0.3)$ & $0.793^{*}$ \\
\hline Warfarin & $84(3.3)$ & $8(0.8)$ & $840(55.7)$ & $31(22.5)$ & $169(52.8)$ & $57(12.5)$ & $31(4.5)$ & $<0.001^{*}$ \\
\hline Statin & 2,267 (89.8) & $848(88.1)$ & $1,085(73.9)$ & $96(69.6)$ & $250(79.9)$ & $393(86.4)$ & $539(79.5)$ & $<0.001^{*}$ \\
\hline
\end{tabular}

Values are number of patients (\%), unless otherwise noted.

${ }^{*}$ Chi-square test; ${ }^{\dagger}$ ANOVA test; ${ }^{\ddagger}$ Kruskal-Wallis test.

LAA, large artery atherosclerosis; SVO, small vessel occlusion; CE, cardioembolism; OD, other determined cause; UD, undetermined cause; FAT, first abnormal time; NIHSS, National Institutes of Health stroke scale; SD, standard deviation; IQR, interquartile range; mRS, modified Rankin Scale; FBS, fasting blood sugar; SBP, systolic blood pressure; DBP, diastolic blood pressure; IV, intravenous; IA, intra-arterial. 
tory or ECG identifies high-risk cardioembolic sources with coexistence of relevant stenosis, then that infarction is classified as "UD $\geq 2$." When there is evidence of chronic occlusion, or no or low risk cardioembolic source with relevant stenosis, it is classified as "LAA." Occlusion on pre-stroke angiographic evaluation, border zone infarction with clinical settings suggestive of hemodynamic failure, or recent (within 1 month of stroke onset) transient ischemic attack corresponding to occlusion site is considered as evidence for chronic occlusion.

\section{Step 4. Consideration of recanalization status of occluded artery after thrombolytic therapy (Figure 1D)}

If there is occlusion but no evidence of chronic occlusion and thrombolytic therapy, including mechanical thrombectomy, is performed, then the recanalization status after thrombolytic therapy should be considered. When residual stenosis exists, or angioplasty or stenting is performed for atherosclerotic stenoocclusion, the underlying vascular pathology is considered primarily as atherosclerotic. When occlusion is resolved completely, comprehensive cardioembolic work-up is recommended. In this situation, low-risk cardioembolic sources are regarded as explaining the etiology of stroke.

\section{Step 5. Consideration of follow-up recanalization status of occluded artery without thrombolytic therapy (Figure 1E)}

When there is occlusion and thrombolytic therapy is not performed, follow-up angiographic evaluation is recommended and recanalization status on that evaluation guides further investigation and determination of stroke subtypes.

\section{Assessment of stroke recurrence}

During hospitalization and up to one year from stroke onset after discharge, stroke recurrence was captured prospectively by dedicated and trained stroke nurses through review of medical records, and direct or telephone interview. Monthly review of event data quality and adjudication of events were performed by the independent outcome adjudication committee.

Within 21 days of the index stroke, recurrent stroke or any neurologic worsening after a period of neurological stability or improvement lasting at least $24 \mathrm{~h}^{13}$ and attributable to new discrete lesions on follow-up CT or DWI was defined as a new neurological symptom/sign. After 21 days from the index stroke, recurrent stroke was defined as suddenly developed focal neurological deficits attributable to occlusion or rupture of cerebral vessels and lasting $24 \mathrm{~h}$ or more. Both ischemic and hemorrhagic strokes were considered as recurrent strokes.

\section{Validation of MAGIC}

We validated MAGIC by comparing the inter-rater reliability of subtype classification with and without applying MAGIC. Forty patients with acute ischemic stroke were randomly selected among those registered in the CRCS-5 database before 2011. Five stroke physicians and five neurology residents from 10 participating centers took part in this validation study (Supplementary Figure 1). The intra-class correlation coefficient (ICC) was obtained to assess intra-rater reliability.

\section{Statistical methods}

Values are presented as means $\pm \mathrm{SD}$ or median (interquartile range $[\mathrm{IQR}]$ ) for continuous variables and as the number (\%) of subjects for categorical variables.

Baseline characteristics (Table 1) and extent of diagnostic evaluation (Table 2) were compared according to stroke subtypes using analysis of variance or Kruskal-Wallis test for con-

Table 2. The extent of diagnostic evaluation according to stroke subtypes

\begin{tabular}{|c|c|c|c|c|c|c|c|c|}
\hline & $\begin{array}{c}\text { LAA } \\
n=2,541\end{array}$ & $\begin{array}{c}\text { SVO } \\
n=964\end{array}$ & $\begin{array}{c}\text { CE } \\
n=1,508\end{array}$ & $\begin{array}{c}\text { OD } \\
n=138\end{array}$ & $\begin{array}{c}\text { UD } \\
\text { Two or more } \\
n=320\end{array}$ & $\begin{array}{c}\text { UD } \\
\text { Negative } \\
n=457\end{array}$ & $\begin{array}{c}\text { UD Incomplete } \\
n=696\end{array}$ & $P$ value ${ }^{*}$ \\
\hline Brain $\mathrm{MRA}^{\dagger}$ & $2,217(87.2)$ & 834 (86.5) & $1,287(85.3)$ & $113(81.9)$ & $267(83.4)$ & $414(90.6)$ & $539(77.4)$ & $<0.001$ \\
\hline CT angiography & 1,003 (39.5) & $314(32.6)$ & $634(42.0)$ & $54(39.1)$ & $128(40.0)$ & $172(37.6)$ & $299(43.0)$ & $<0.001$ \\
\hline Conventional angiography & $465(18.3)$ & $23(2.4)$ & $21(13.9)$ & $36(26.1)$ & $38(11.9)$ & $67(14.7)$ & $32(4.6)$ & $<0.001$ \\
\hline Transcranial Doppler & $1,883(74.1)$ & $748(77.6)$ & $973(64.5)$ & $89(64.5)$ & $215(67.2)$ & $388(84.9)$ & $368(52.9)$ & $<0.001$ \\
\hline Brain SPECT & $130(5.1)$ & $9(0.9)$ & $18(1.2)$ & $4(2.9)$ & $9(2.8)$ & $7(1.5)$ & $10(1.4)$ & $<0.001$ \\
\hline Transthoracic echocardiogram & $1,695(66.7)$ & $671(69.6)$ & $998(66.2)$ & $83(60.1)$ & $230(71.9)$ & $370(81.0)$ & $365(52.4)$ & $<0.001$ \\
\hline Transesophageal echocardiogram & $360(14.2)$ & $117(12.1)$ & $243(16.1)$ & $23(16.7)$ & $38(11.9)$ & $160(35.0)$ & $27(3.9)$ & $<0.001$ \\
\hline 24-h Holter monitoring & $853(33.6)$ & $260(27.0)$ & $430(28.5)$ & $45(32.6)$ & $76(23.8)$ & $287(62.8)$ & $197(28.3)$ & $<0.001$ \\
\hline Extensive embolic source evaluation ${ }^{\ddagger}$ & $325(12.8)$ & $98(10.2)$ & $196(13.0)$ & $21(15.2)$ & $27(8.4)$ & $141(30.9)$ & $21(3.0)$ & $<0.001$ \\
\hline
\end{tabular}

Values are number of patients (\%). See Table 1 for abbreviations.

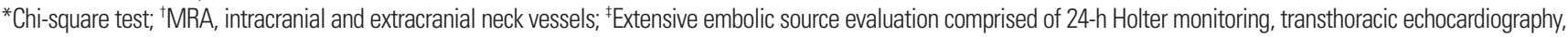
and transesophageal echocardiography during hospital stay.

MRA, magnetic resonance angiography; CT, computed tomography; SPECT, single-photon emission computed tomography 
tinuous variables and Pearson's chi-squared test for categorical variables. Stroke recurrence rates were estimated using the Kaplan-Meier product-limit method. Cumulative 7-day, 30-day, 90-day, and 1-year rates of stroke recurrence and their $95 \%$ confidence intervals (CIs) were calculated for the entire study population and according to stroke subtypes. All statistical analyses were performed with SPSS (version 18.0; SPSS Inc., Chicago, IL, USA), and a 2 -sided $P$ value $<0.05$ was considered as the minimum level of statistical significance.

\section{Results}

In the validation study (Supplementary Figure 1), the overall ICC value was 0.43 (95\% CI, 0.31-0.57) for subtype classification with MAGIC and 0.28 (95\% CI, 0.18-0.42) for classification without MAGIC. Among the stroke physicians, the ICC was 0.56 (95\% CI, 0.42-0.70) with MAGIC and 0.35 (95\% CI, 0.21-0.51) without MAGIC. Among the neurology residents, the ICC was 0.37 (95\% CI, 0.23-0.54) with MAGIC and 0.25 (95\% CI, 0.13-0.42) without MAGIC.

During the study period, 11,652 patients with acute ischemic stroke were admitted to the participating hospitals within 7 days after stroke onset. After excluding 1,876 patients due to absence of acute ischemic lesions in DWI or unavailable DWI, 3,092 patients due to unavailability of MAGIC data, and another 60 patients lacking information on stroke recurrence, a total of 6,624 patients were enrolled in this study.

Baseline characteristics according to ischemic stroke subtypes are presented in Table 1 . The proportion of LAA subtype was the highest (38.3\%) followed by CE (22.8\%), UD (22.2\%; UD $\geq 2,4.8 \%$, UD-negative, $6.9 \%$, UD incomplete, $10.5 \%$ ), SVO (14.6\%), and OD (2.1\%). The CE subtype was found in the oldest patients (mean \pm SD, $71.9 \pm 11.6$ years), had the shortest onset to arrival time (median, 3 hours) and showed the most severe presenting symptoms (median National Institute of Health Stroke Scale (NIHSS), 8) compared to the other subtypes. SVO showed the lowest frequency of previous stroke history (15.8\%), but interestingly the highest frequency of current smoking (47.2\%), and the highest systolic or diastolic blood pressure.

Diabetes mellitus, hyperlipidemia, and total cholesterol level, were more frequent in LAA than in CE or SVO subtypes. Coronary artery disease and atrial fibrillation were most prevalent in $\mathrm{CE}$ and $\mathrm{UD} \geq 2$. Thrombolytic therapies were most frequently performed in CE (30.4\%) followed by UD $\geq 2(22.2 \%)$ and UD-negative (19.0\%). Aspirin (70.7\%) and clopidogrel (33.9\%) were the most frequently prescribed antithrombotics at discharge, and statin was administered to more than $80 \%$ of patients at discharge. The prescription rate was the highest in LAA (89.8\%)

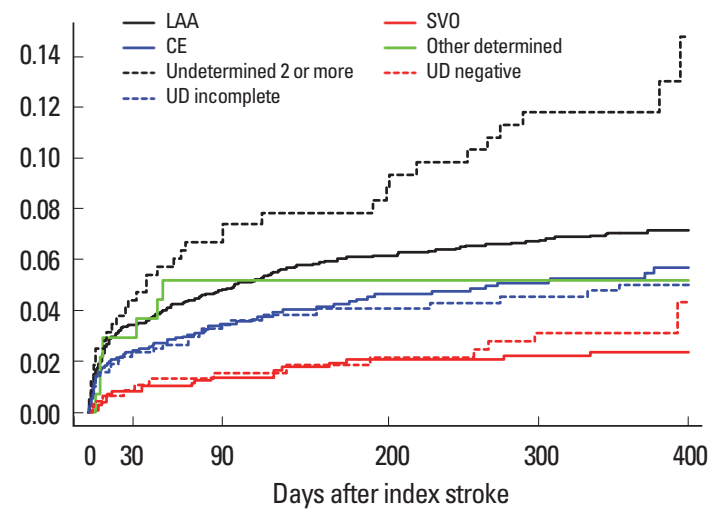

\begin{tabular}{|c|c|c|c|c|c|c|}
\hline LAA & 2,531 & & 2,225 & 1,706 & 1,689 & \\
\hline SVO & 963 & 942 & 876 & 669 & 666 & 150 \\
\hline CE & 1,500 & 1,411 & 1,265 & 933 & 918 & 228 \\
\hline $\begin{array}{l}\text { Other determined } \\
\text {. }\end{array}$ & 136 & 130 & 120 & 96 & 95 & 17 \\
\hline Jndetermined 2 or more & 319 & 297 & 261 & 185 & 178 & 37 \\
\hline $\begin{array}{l}\text { UD negative } \\
\text { Uncomplete }\end{array}$ & $45 /$ & ${ }_{6}^{445}$ & $\begin{array}{l}404 \\
583\end{array}$ & 315 & 311 & 61 \\
\hline UD incomplete & & 636 & 583 & & 404 & 107 \\
\hline
\end{tabular}

Figure 2. Kaplan-Meier survival curves comparing risk of recurrent stroke. Cumulative analysis shows fatal or nonfatal recurrent stroke rates according to stroke subtypes. LAA, large artery atherosclerosis; SVO, small vessel occlusion; $\mathrm{CE}$, cardioembolism; UD, undetermined cause.

followed by SVO (88.1\%). Many of the patients with UD $\geq 2$ had both characteristics of patients with CE and LAA; UD $\geq 2$ were more likely to be older, have stroke history, hypertension, diabetes mellitus, hyperlipidemia, atrial fibrillation, coronary artery disease, and more severe neurologic deficits.

Table 2 presents the extent of diagnostic evaluation according to stroke subtypes. All subjects underwent DWI and most patients across all subtypes underwent magnetic resonance angiography (MRA) (77\%-90\%). Extensive embolic source evaluation was performed on an average of $12.5 \%$ of patients, and the highest rate was for UD-negative among all stroke subtypes (30.9\%).

Stroke recurrence rates during the first year were as follows: $1.7 \%$ (95\% CI, $1.3 \%-2.1 \%)$ at 1 week, $2.8 \%(2.4 \%-3.2 \%)$ at 1 month, $4 \%(3.6 \%-4.4 \%)$ at 3 months, and $5.9 \%$ (5.3\%-6.5\%) at 1 year. One-year recurrence rates were the highest for UD $\geq 2$ (11.8\%) followed by LAA (7.3\%), OD (5.9\%), CE (5.6\%), UD incomplete (5.4\%), UD-negative (3.1\%), and SVO (2.5\%) (Figure 2).

MAGIC has three special categories in addition to TOAST subtypes: LAA-BR, LAA-NG, and LAA-LC. Table 3 shows clinical characteristics of special categories compared with classically defined LAA (pure LAA) and SVO. With respect to clinical characteristics, LAA-BR and LAA-LC were similar to pure LAA, and LAA-NG was similar to SVO. One-year stroke recurrence rates were highest in pure LAA $(8.3 \%)$ followed by LAALC (6.2\%), LAA-NG (4.2\%), LAA-BR (3.7\%), and SVO (2.5\%) (Figure 3). 
Table 3. Baseline characteristics according to special categories of MAGIC

\begin{tabular}{|c|c|c|c|c|c|c|}
\hline & $\begin{array}{c}\text { Pure-LAA } \\
n=1,837\end{array}$ & $\begin{array}{l}\text { LAA-LC } \\
n=328\end{array}$ & $\begin{array}{l}\text { LAA-BR } \\
n=267\end{array}$ & $\begin{array}{c}\begin{array}{c}\text { LAA-NG } \\
n=145\end{array}\end{array}$ & $\begin{array}{c}\text { SVO } \\
n=956\end{array}$ & $P$-value \\
\hline Male & $1,158(63.0)$ & $183(55.8)$ & $134(50.2)$ & 109 (75.2) & $594(62.1)$ & $<0.001^{*}$ \\
\hline Age (years), mean \pm SD & $68.2 \pm 12.2$ & $68.6 \pm 12.2$ & $67.0 \pm 10.8$ & $61.3 \pm 12.7$ & $63.9 \pm 12.7$ & $<0.001^{\dagger}$ \\
\hline FAT to arrival (h), median (IOR) & $10(2-46)$ & $15(4-39)$ & $17(5-41)$ & $15(4-45)$ & $14(4-40)$ & $<0.001^{\ddagger}$ \\
\hline Initial NIHSS, median (IOR) & $4(2-8)$ & $3(1-5)$ & $3(2-5)$ & $2(1-4)$ & $2(1-4)$ & $<0.001^{\ddagger}$ \\
\hline History of stroke & $428(23.3)$ & $81(24.7)$ & $52(19.5)$ & $19(13.1)$ & $150(15.7)$ & $<0.001^{*}$ \\
\hline Coronary artery disease & $133(7.2)$ & $18(5.5)$ & $14(5.2)$ & $8(5.5)$ & $50(5.2)$ & $<0.001^{*}$ \\
\hline Hypertension & $1278(69.6)$ & $254(77.4)$ & $187(70.0)$ & $95(65.5)$ & $624(65.3)$ & $<0.001^{*}$ \\
\hline Diabetes mellitus & $668(36.4)$ & $140(42.7)$ & $120(44.9)$ & $57(39.3)$ & $321(33.6)$ & $<0.001^{*}$ \\
\hline Hyperlipidemia & 640 (34.8) & $107(32.6)$ & $84(31.5)$ & $42(29.0)$ & $305(31.9)$ & $<0.001^{*}$ \\
\hline Current smoking & $809(44.0)$ & $115(35.1)$ & $86(32.2)$ & $74(51.0)$ & $452(47.3)$ & $<0.001^{*}$ \\
\hline Thrombolysis & & & & & & $<0.001^{*}$ \\
\hline IV & $152(8.3)$ & $14(4.3)$ & $18(6.7)$ & $6(4.1)$ & $49(5.1)$ & \\
\hline $\mathrm{IA}$ & $58(3.2)$ & $5(1.5)$ & $0(0)$ & $1(0.7)$ & $0(0)$ & \\
\hline IV+IA & 60 (3.3) & $5(1.5)$ & $0(0)$ & $0(0)$ & $0(0)$ & \\
\hline
\end{tabular}

Values are number of patients (\%), unless otherwise noted. See Tables 1 and 2 for abbreviations.

${ }^{*}$ Chi-square test; ${ }^{\dagger} \mathrm{ANOV} A$ test; ${ }^{\ddagger}$ Kruskal-Wallis test.

Pure-LAA, pure large artery atherosclerosis; LAA-BR, large artery atherosclerosis with branch atheromatous disease; LAA-NG, large artery atherosclerosis with normal angiography; LAA-LC, large artery atherosclerosis with lacunae.

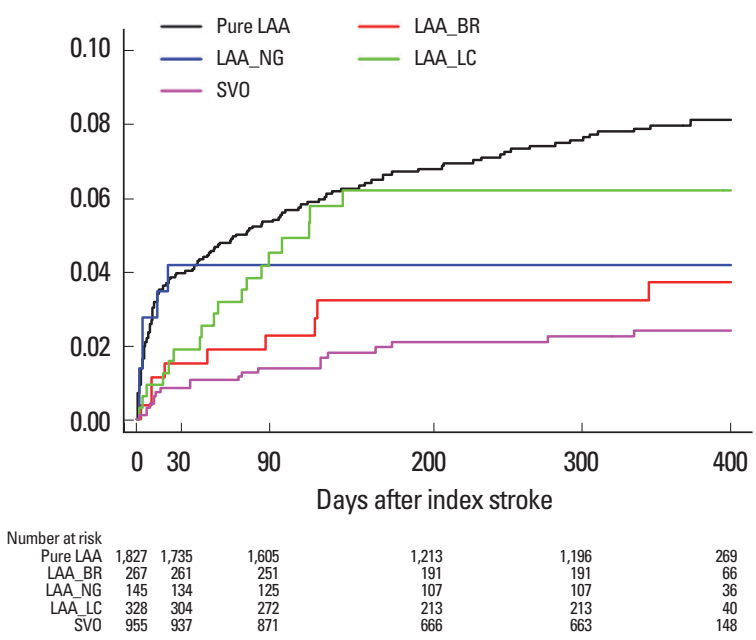

Figure 3. Kaplan-Meier survival curves comparing risk of recurrent stroke. Cumulative analysis shows fatal or nonfatal recurrent stroke rates according to special categories of MAGIC. LAA, large artery atherosclerosis; Pure LAA, classically defined $L A A$; $L A A-B R$, branch atheromatous disease; $L A A-N G$, LAA with normal angiography; LAA-LC, LAA with lacunae; SVO, small vessel occlusion.

\section{Discussion}

TOAST classification was used widely in practice and research from the 1990s before the use of MRI in patients with acute stroke became popular. However, TOAST's major weakness is that the proportion of patients whose stroke mechanisms are undiagnosed (UD) is quite high, ${ }^{14}$ which may be attributed to the underdevelopment of diagnostic technologies specific to stroke mechanisms, ${ }^{15}$ as well as confusion in determining stroke subtypes in patients who are treated with thrombolytic therapy. With the hope of improving inter-rater reliability and reducing the proportion of UD, MAGIC is an attempt to diagnose stroke mechanisms, keeping in mind the rapidly developing MR technologies and wider use of thrombolytic therapy.

Compared to previous studies, the use of MAGIC did not improve the inter-rater reliability of subtype classification. However, we observed a modest improvement of ICC with MAGIC in the validation study population. ${ }^{1,2}$ The increase of ICC with MAGIC may be attributed to the algorithm and reduced ambiguity with 3 special categories (LAA-LC, LAA-BR, and LAA$\mathrm{NG}$ ), potentially enabling most physicians to ascribe more consistent stroke subtypes.

Contrary to our expectations, applying MAGIC could not reduce the proportion of UD (22.2\% vs. $16.2 \%-40.6 \%) .{ }^{16-18} \mathrm{~A}$ decrease of UD-negative by extensive etiologic evaluation seemed to be offset by an increase of UD incomplete due to a stringent requirement of extensive evaluation in patients possibly with an embolic stroke. $^{18,19}$

The proportions of stroke subtypes by MAGIC other than UD were not different from those in other hospital-based stroke registries except for $\mathrm{CE}$, which showed relatively higher frequency (LAA [38.3\% vs. $16.5 \%-57.9 \%$ ], SVO [14.6\% vs. $14.8 \%-34.7 \%$ ], CE [22.8\% vs. $8.7 \%-18.3 \%]$, and OD [2.1\% vs. $3.1 \%-7.0 \%]) .{ }^{16-18}$ However, the proportions of stroke subtypes in community-based studies were different with LAA being less common (9.3\%$20.9 \%)$, and SVO (20.5\%-27.0\%) and CE (25.6\%-30.2\%) being more common. ${ }^{19-21}$

Comparisons of baseline characteristics according to stroke subtypes (Table 1) confirm our previous work: LAA is more tightly associated with risk factors of atherosclerosis, and patients with $\mathrm{CE}$ are more likely to be older and female and have more 
severe neurologic deficits. ${ }^{16,20,21}$ Despite relatively low frequency of diabetes mellitus in $\mathrm{CE}$, that $\mathrm{CE}$ is associated with the highest fasting blood sugar levels may be due to stress-induced hyperglycemia related with more severe neurologic deficits than other subtypes.

MAGIC recommended extensive embolic source evaluation in cases with non-lacunar infarction and no arterial pathology in relevant arteries. Furthermore, using new imaging techniques such as DWI and high-resolution wall imaging, the definition of relevant arterial pathology was extended to stenosis in less than $50 \%$ of patients. As a result, the proportion of UD-negative was reduced to $6.9 \%$, which is substantially lower than those reported by previous studies (17.5\%-24.2\%). ${ }^{19,21,22}$ It should be noted that in this study, less than a third of patients underwent extensive embolic source evaluation, even in the UD-negative group. More active application of extensive embolic source evaluation and other advanced technologies may lead to further reduction of UD-negative classifications. ${ }^{15}$

One-year stroke recurrence rate was highest for $\mathrm{UD} \geq 2(11.8 \%)$ (Figure 2), which may be explained by the fact that UD $\geq 2$ had characteristics of both LAA and CE, the riskiest profiles with respect to stroke recurrence. Most previous studies reported high risk of recurrence in LAA or UD. ${ }^{6,13,20}$ Recurrence rate of LAA was the second highest in this study (7.3\%). The low recurrence rate of UD-negative (3.1\% at one year) must also be noted. A previous study reported that stroke recurrence rate was highest for UD-negative followed by UD $\geq 2$ and LAA. ${ }^{6}$ However, in that study, the proportions of cases of UD-negative and CE were $18.1 \%$ and $10.8 \%$, respectively, and the discrepancies with respect to the proportions of UD-negative and CE in our current study are probably due to the difference in the extent of embolic source evaluation. The extensive embolic source evaluation in our study may explain the lower rate of recurrence for UD-negative by exclusion of high-risk $\mathrm{CE}$ from this subgroup. All recurrences in OD occurred within 3 months of onset, in line with the natural history of arterial dissection, the most common cause of $\mathrm{OD}^{23}$

LAA-LC and LAA-BR have a single small lesion on DWI like SVO but their clinical profiles, such as age, risk factors of atherosclerosis, and history of stroke were similar to that of pure LAA (Table 3 ) and their one-year recurrence rates $(6.2 \%$ and $3.7 \%$, respectively) were between those of pure LAA and SVO (8.3\% and $2.5 \%$, respectively) (Figure 3). High-risk profiles of LAA-LC may be attributed to the coexistent LAA. In the design stage of this study, we expected LAA-NG to be similar to LAA. On the contrary, the study results revealed that LAA-NG was clinically similar to SVO and its recurrence rate was slightly higher than SVO (4.2\% vs. $2.5 \%$ ). The recurrence rate of LAA-
BR (3.7\%) was between that of SVO and LAA-NG. Previously, LAA-BR was reported as a subtype of LAA with high risk of recurrence. $^{22}$

Limitations of this study should be noted. First, despite all effort, the proportion of cases of UD was not reduced, although the proportion of cases of UD-negative seemed to be decreased. The retrospective nature of subtype classification, namely determining subtypes after discharge through regular registry meetings, may contribute to the high proportion of UD. Second, MAGIC was only a recommendation to the participating physicians; we only provided a useful algorithm for determining stroke subtypes, and the stroke physicians themselves decided whether to adopt it. We are developing a smart phone application for MAGIC, which is expected to improve the feasibility and applicability of this algorithm. Third, several definitions used in MAGIC such as LAA-NG, LAA-BR, and LAA-LC were somewhat arbitrary. Their clinical meanings should be determined by further research. Forth, despite its uniqueness and applicability, previous studies do not support the consideration of results of recanalization therapy in determining stroke mechanisms. Lastly, that this was a multicenter stroke registry-based observational study limits the representativeness and generalizability of the study results, although application of MAGIC was performed prospectively.

\section{Conclusions}

This study shows that our MRI-based diagnostic algorithm for assisting TOAST classification, which incorporates recent advances in stroke imaging and recanalization therapies, is feasible and may improve the consistency of subtype classification in clinical practice. However, we encountered several problems, such as a lower inter-rater reliability than expected, no reduction of undetermined etiology, and arbitrariness of adopted definitions. Future research including development of an application tool for the algorithm is warranted.

\section{References}

1. Ay H, Furie KL, Singhal A, Smith WS, Sorensen AG, Koroshetz WJ. An evidence-based causative classification system for acute ischemic stroke. Ann Neurol 2005;58:688-697.

2. Meschia JF, Barrett KM, Chukwudelunzu F, Brown WM, Case LD, Kissela BM, et al. Interobserver agreement in the trial of org 10172 in acute stroke treatment classification of stroke based on retrospective medical record review. J Stroke Cerebrovasc Dis 2006; 15:266-272.

3. Adams HP, Bendixen BH, Kappelle LJ, Biller J, Love BB, Gor- 
don DL, et al. Classification of subtype of acute ischemic stroke. Definitions for use in a multicenter clinical trial. TOAST. Trial of Org 10172 in Acute Stroke Treatment. Stroke 1993;24:3541.

4. Gordon DL, Bendixen BH, Adams HP, Clarke W, Kappelle LJ, Woolson RF. Interphysician agreement in the diagnosis of subtypes of acute ischemic stroke: implications for clinical trials. The TOAST Investigators. Neurology 1993;43:1021-1027.

5. Amarenco P, Bogousslavsky J, Caplan LR, Donnan GA, Hennerici MG. Classification of stroke subtypes. Cerebrovasc Dis 2009; 27:493-501.

6. Bang OY, Lee PH, Joo SY, Lee JS, Joo IS, Huh K. Frequency and mechanisms of stroke recurrence after cryptogenic stroke. Ann Neurol 2003;54:227-234.

7. Kim BJ, Han MK, Park TH, Park SS, Lee KB, Lee BC, et al. Current status of acute stroke management in Korea: a report on a multicenter, comprehensive acute stroke registry. Int J Stroke 2014;9:514-518.

8. Ko SB, Choi SI, Chun EJ, Ko Y, Park JH, Lee SJ, et al. Role of cardiac multidetector computed tomography in acute ischemic stroke: a preliminary report. Cerebrovasc Dis 2010;29:313-320.

9. Amarenco P, Hauw JJ, Gautier JC. Arterial pathology in cerebellar infarction. Stroke 1990;21:1299-1305.

10. Amarenco P, Rosengart A, DeWitt LD, Pessin MS, Caplan LR. Anterior inferior cerebellar artery territory infarcts. Mechanisms and clinical features. Arch Neurol 1993;50:154-161.

11. Levy R, Duyckaerts C, Hauw JJ. Massive infarcts involving the territory of the anterior choroidal artery and cardioembolism. Stroke 1995;26:609-613.

12. Kim K, Lee HS, Jung YH, Kim YD, Nam HS, Nam CM, et al. Mechanism of medullary infarction based on arterial territory involvement. J Clin Neurol 2012;8:116-122.

13. Petty GW, Brown RD, Jr., Whisnant JP, Sicks JD, O’Fallon WM, Wiebers DO. Ischemic stroke subtypes: a population-based study of functional outcome, survival, and recurrence. Stroke 2000;31:1062-1068.

14. Marnane M, Duggan CA, Sheehan OC, Merwick A, Hannon $\mathrm{N}$, Curtin D, et al. Stroke subtype classification to mechanism- specific and undetermined categories by TOAST, A-S-C-O, and causative classification system: direct comparison in the North Dublin population stroke study. Stroke 2010;41:15791586.

15. Bang OY, Ovbiagele B, Kim JS. Evaluation of cryptogenic stroke with advanced diagnostic techniques. Stroke 2014;45:11861194.

16. Deleu D, Inshasi J, Akhtar N, Ali J, Vurgese T, Ali S, et al. Risk factors, management and outcome of subtypes of ischemic stroke: a stroke registry from the Arabian Gulf.J Neurol Sci 2011; 300:142-147.

17. Lee BI, Nam HS, Heo JH, Kim DI, Yonsei Stroke Team. Yonsei Stroke Registry. Analysis of 1,000 patients with acute cerebral infarctions. Cerebrovasc Dis 2001;12:145-151.

18. Shang W, Liu J. Stroke subtype classification: a comparative study of ASCO and modified TOAST. J Neurol Sci 2012;314: 66-70.

19. Hajat C, Heuschmann PU, Coshall C, Padayachee S, Chambers J, Rudd AG, et al. Incidence of aetiological subtypes of stroke in a multi-ethnic population based study: the South London Stroke Register. J Neurol Neurosurg Psychiatry 2011;82:527533.

20. Kolominsky-Rabas PL, Weber M, Gefeller O, Neundoerfer B, Heuschmann PU. Epidemiology of ischemic stroke subtypes according to TOAST criteria: incidence, recurrence, and longterm survival in ischemic stroke subtypes: a population-based study. Stroke 2001;32:2735-2740.

21. Grau AJ, Weimar C, Buggle F, Heinrich A, Goertler M, Neumaier $S$, et al. Risk factors, outcome, and treatment in subtypes of ischemic stroke: the German stroke data bank. Stroke 2001; 32:2559-2566.

22. Kim BJ, Kim JS. Ischemic Stroke Subtype Classification: An Asian Viewpoint. J Stroke 2014;16:8-17.

23. Yesilot Barlas N, Putaala J, Waje-Andreassen U, Vassilopoulou S, Nardi K, Odier C, et al. Etiology of first-ever ischaemic stroke in European young adults: the 15 cities young stroke study. Eur J Neurol 2013;20:1431-1439. 
Supplementary Table 1. Other explainable etiology (non-cardioembolic, non-atherothromboembolic, or non-lacunar cause) of acute ischemic stroke

Abnormalities of thrombosis and hemostasis

Acute arterial dissection

Acute disseminated intravascular coagulation

CADASIL (Cerebral autosomal-dominant arteriopathy with subcortical infarcts and leukoencephalopathy cerebral vasculitis)

Cerebral venous thrombosis

Chronic arterial dissection

Clinically relevant aneurysm

Drug-induced stroke

Fibromuscular dysplasia

Heparin-induced thrombocytopenia type II

Hyperviscosity syndromes

Hypoperfusion syndromes

latrogenic causes

Meningitis

Migraine-induced stroke

MELAS (Mitochondrial encephalomyopathy with lactic acidosis and stroke-like episodes)

Moyamoya disease

Primary antiphospholipid antibody syndrome

Primary infection of the arterial wall

Segmental vasoconstriction or vasospasm

Sickle cell disease

Sneddon syndrome

Thrombotic thrombocytopenic purpura - hemolytic uremic syndrome 
Supplementary Table 2. Cardioembolic sources of ischemic stroke

Sources of high primary risk of stroke

Left atrial thrombus

Left ventricular thrombus

Atrial fibrillation

Paroxysmal atrial fibrillation

Sick sinus syndrome

Atrial flutter

Recent myocardial infarction ( $<4$ weeks)

Rheumatic mitral or aortic valve disease

Bioprosthetic or mechanical heart valves

Chronic myocardial infarction with ejection fraction $<28 \%$

Congestive heart failure with ejection fraction $<30 \%$

Dilated cardiomyopathy

Nonbacterial thrombotic endocarditis

Infective endocarditis

Papillary fibroelastoma

Left atrial myxoma

Sources of low or uncertain primary risk of stroke

Mitral annulus calcification

Patent foramen ovale

Atrial septal aneurysm

Atrial septal aneurysm and patent foramen ovale

Ventricular aneurysm without thrombus

Isolated left atrial smoke

Complex atheroma in the ascending or proximal arch 
Supplementary Figure 1. Validation study of MRI-based algorithm for acute ischemic stroke classification (MAGIC).

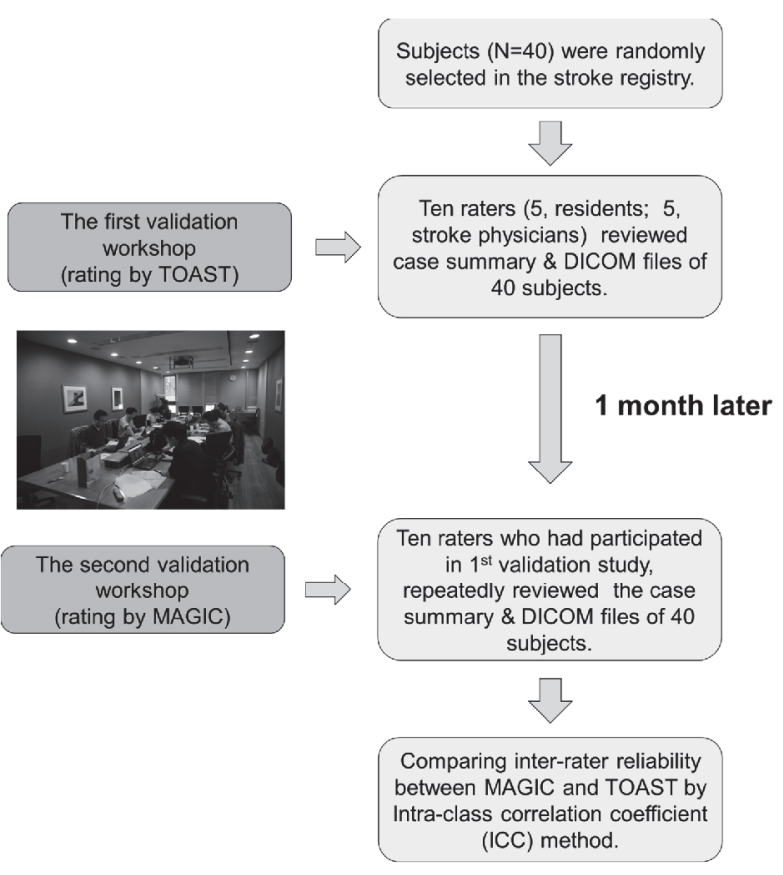

